\title{
Usage Pattern of the Complex Masticatory Muscles in the Shingleback Lizard, Trachydosaurus rugosus: A Model for Muscle Placement
}

CARL GANS, FRITS DE VREE, AND DAVID CARRIER

Division of Biological Sciences, The University of Michigan, Ann Arbor, Michigan 48109-1048 (C.G., D.C.) and The Department of Biology, The University of Antwerp (UIA), 2610 Wilrijk, Antwerp, Belgium (F.d.V.)

\begin{abstract}
This wide-ranging, omnivorous lizard of Australia has a very complex adductor muscle mass, with fibers differing in length by a factor of three and in insertion angle by $90^{\circ}$. Stimulated muscles produce maximal moment with the mouth nearly fully open. The opening mechanism appears to involve only simple rotation and no translation of the mandible. EMGs indicate that the entire mass is activated equivalently in crushing and there are no temporal subdivisions, for instance, matching activity to angle of opening. During crushing of hard objects, the chin is brought into contact with the ground so that the subvertebral muscles may aid buccal closure. The lizards also activate the muscles in a pulsatile staircase effect leading to an unfused tetanus that generates forces several times the twitch level. Application in parallel of a maximum number of sarcomeres to the crushing bite appears to be the major design characteristic. Hence, this species offers an ideal case for analysis of the effects of different sarcomere placements on the simple movement generated. For the primary adductor muscles, the angles of fiber insertion relative to the lines connecting each insertion with the jaw joint are equivalent; this relation persists as the mouth opens. Also, fiber lengths are proportional to the distance between jaw joint and site of insertion so that each sarcomere contributes equally to the movement generated. Complex tendons provide additional space for muscle placement. Some of these also extend beyond the bony attachment sites, producing tendinous "coronoid processes." The fibers of laterally and ventrally placed muscles are short relative to the length of the entire muscle, insert at relatively short moment arms, and undergo short excursion during opening; however, there are many such fibers. Also, muscles with a low incident angle are crossed; they apparently protect the jaw joint from horizontal (disarticulating) forces.
\end{abstract}

The shingleback skink or sleepy lizard, Trachydosaurus rugosus, is a locally common form that ranges clear across Australia and onto offshore islands (Cogger, 1983). Its extensive range and the rather diverse habitats occupied reflect its omnivorous food habits, including "insects and other arthropods, snails, carrion, flowers, fruit and berries" (Cogger, 1983). Shinglebacks apparently take tomatoes in agricultural areas, whereas Western Australian populations feed on the introduced snail, Helix pomacia.

Their size, ease of handling, extremely slow movement, and availability make them ideal for the analysis of mastication. This report is the second of a pair (Wineski and Gans, 1984) analyzing their feeding system. It provides one of a continuing series of studies of motor sequences in the mandibular musculature of reptiles (Gorniak et al., 1982; Gomez and Gasc, 1973; see also Smith, 1982; Throckmorton, 1978). Dissection shows that Trachydosaurus have a bulky and extremely complex mandibular adductor musculature, but apparently lack all but faint traces of cranial

Received December 27, 1984. Accepted March 11, 1985 
kinesis. This makes them good experimental animals in which to study the problem of fiber placement in the adductor system. We here provide fiber lengths and angles at the open and closed positions for the major adductor muscles and document the usage pattern of the system by electromyography (EMG). The present studies also provided the opportunity to test an inexpensive and portable EMG set-up (Gans and Gorniak, 1982) and to check the results thus obtained against analyses obtained from the same species with more sophisticated equipment available in the laboratory.

\section{MATERIALS AND METHODS}

This study is based on some dozen shingleback lizards, Trachydosaurus rugosus (mass $500-600 \mathrm{gm})$, collected in the vicinity of Perth, Western Australia, and maintained in the laboratory on a diet of lettuce and tomatoes, snails, cat food, insects, and miscellaneous pieces of meat. Ticks found on the animals (particularly in the external auditory meatus) were removed carefully (Bull, 1978), and the lesions were permitted to heal before the animals were used. In Perth, the specimens were kept in open cages in a partially shaded shed until they were needed for experiments; in Ann Arbor they were held on a 12-hr-light, 12-hr-dark cycle individually or as pairs in cages with individual temperature gradients. They were observed and filmed while feeding on lettuce leaves, tomatoes, plums, grapes, canned dog-food, crickets, cockroaches, and snails (Helix pomacia). They were conditioned to be fed by hand, close to the front of a screened Faraday cage fitted with spot heat lamps and sometimes with a $45^{\circ}$ mirror. Control films of feeding on snails and tomatoes were taken for later comparison with those of instrumented animals. These two items represent the extremes of a range of food types (neither Helix nor tomato are native to Australia, although food items equivalent in size and hardness are taken there). We also recorded the threat display, which consists of an extreme forward and upwardly directed gape during which the head is elevated on the neck (Fig. 3F). Simple feeding sequences (without metal implants) were tested by cinefluoroscopy, in the Antwerp laboratory of Dr. de Vree.

The breaking strength of some 20 snails, in various directions relative to their spiral axis, was tested in a simple static loading system. Each snail was covered with a single layer of Saran wrap and one surface was embedded in a petri dish filled to a depth of $8 \mathrm{~mm}$ with plaster of paris. After curing for $24 \mathrm{hr}$ each dish was positioned on a platform, and force was slowly applied to the free surface (at right angles to the dish). Point contact was avoided by covering the forcetransmitting lever with a thin layer of suede leather. The force was applied gradually by filling a bucket with water.

The following procedures are those for the portable EMG set-up (modifications allowed in the main laboratory are listed in parentheses). Prior to the operation, two patches of Velcro were glued to the back of the animal with neoprene adhesive, one to the osteoderms over the back of the skull and the other to those on the middle of the back, just posterior to the pectoral girdle. In preparation for the insertion of electrodes, animals were anesthetized by intraperitoneal injection of $10-15 \mathrm{mg}$ per $\mathrm{kg}$ of body mass of Brietal Sodium (methohexitone sodium, or Brevital in U.S.) in saline (or intramuscular injection of $100 \mathrm{mg}$ per $\mathrm{kg}$ of Ketamine). A small dental burr, fitted with a depth collar exposing 1 to $2 \mathrm{~mm}$ of the bit, was then used to perforate the osteoderms without drilling into the muscle.

The electrodes consisted of two 0.003-inch, stainless-steel, Teflon-coated wires (Medwire) twisted together with $1 \mathrm{~mm}$ of the ends recurved and bared, and the bend reinforced with a drop of nail polish. They were inserted stereotactically with chamfered Intramed No. 22 hypodermic needles. The wires were bent at the surface of the skin. The drilled hole was then plugged with a layer of nail polish, and the wires were passed among the scales to the top of the head. The wires were glued to the skin with neoprene adhesive.

Two harnesses were used, each consisting of a set of $15-\mathrm{cm}$-long flexible cables attached to a gold minipin array (eight or 16 pins for four or eight electrodes) that was mounted in a connector. A matching connector provided the terminal for a set of shielded cables equipped with minipin plugs leading to the amplifiers. The flexible cables were firmly bonded to a piece of Velcro. The free ends of the cables were soldered to the electrode wires and the individual joints then insulated with tape or shrink tubing. The patch of Velcro on the back of the lizard matched that on the harness, thus relieving strain on the electrodes and solder joints. The patch of 
Velcro glued to the top of the head bore a base for a long, narrow strip of Velcro that was looped loosely around the anterior portions of the harness to hold the electrode connections in place and prevent them from being scraped off accidentally. The cable connectors were disconnected between experiments; the connector of the harness then was suspended from the top of the cage by a thin string that would twist more easily than the wires as the animals walked about. Combinations of any four signals from the eight implanted electrodes were preamplified and recorded simultaneously (up to 12 signals could be recorded simultaneously); the remainder were connected to ground.

The recording equipment was limited in the number of channels (but not in their resolution) in order to make it movable. It consisted of four Grass P-15 differential preamplifiers feeding into a modified Sony TC-7884 quadraphonic tape recorder; the high-impedance input connections of the recorder had been changed to panel mount BNC plugs. (Sets of Tektronix 122 and 26A2 preamplifiers were connected to a bank of Honeywell Acudata 117 amplifiers and recorded on a 14-channel medium-bandpass Honeywell 5600 FM tape recorder.) Lowimpedance inputs to each of the four recording channels allowed the records to be cued by microphone signals, thus characterizing particular runs. Connection of preamplifiers, oscilloscope, and chart recorder was by double-crimped BNC cables. A third set of four cables permitted selected monitoring on a two-channel, split-beam oscilloscope of any two channels, either of the input or of the output of the tape recorder between or during recording sessions. (Signals were monitored on a Tektronix 565 dual-beam oscilloscope with twin 3A74 plug-ins, permitting simultaneous observation of eight signals.)

During recording sessions, the front of the Faraday cage could be opened without inducing significant electronic noise. The animals were filmed at $24 \mathrm{fps}$ on Kodachrome 40 Super-8 film with a Braun Nizo 801-macro camera, fitted with a No. 1 close-up lens, while the field was illuminated by a Braun 1,000-W Photolight (or banks of other photoflood bulbs). The strobe contact of the camera was used to trigger a Strobex 122 Lamp, the output of which was monitored by a photocell and recorded on one of the four (14) tape channels.
The quarter-inch tapes recorded in Australia were returned to the U.S., where the output was hard-printed on a Brush eightchannel recorder and also analyzed by a minicomputer system that records the number of EMG spikes and the mean spike amplitude corresponding to each frame of the cine record or any other suitable interval (Beach et al., 1982).

Recordings were of four (eight to 12) muscles simultaneously. Muscles studied and placement of electrodes (checked by postmortem dissection) were as follows (nomenclature after Wineski and Gans, 1984; see present Figure 1 for the layered structure and Figure 2 for the effect of changed fiber angle with opening):

\section{M. adductor mandibulae externus, part 1 (MAME-1), anterior lateral}

In the anteriormost part of the muscle within the set of fibers arising from the medial side of the lateral plate of the quadrate aponeurosis and inserting onto the lateral aspect of the mandible.

\section{MAME-1 posterior}

In the muscle fibers originating from the anteromedial face of the quadrate between the two plates of the quadrate aponeurosis between the medial side of the lateral and from the lateral side of the medial plate of the quadrate aponeurosis. These fibers insert onto the dorsal, lateral, and medial surfaces of the posterior third of the mandible. The insertion of the electrode was via the laterodorsal portion of the postorbital region; the needle was passed in a posteroventral direction until it contacted the anterior face of the quadrate.

\section{MAME-2 anterior lateral}

The needle entered the muscle through the inside of the mouth, passing directly into the muscle in a posterolateral direction for approximately $5 \mathrm{~mm}$. The wires were led off through the angle of the mouth and glued to the labial skin. The wires did not contact the tongue and had no noticeable effect on the feeding behavior.

\section{MAME-2 anterior medial}

The needle entered the muscle through the inside of the mouth, passing directly into the muscle in a dorsal direction for approximately $5 \mathrm{~mm}$. The wires were led off through 

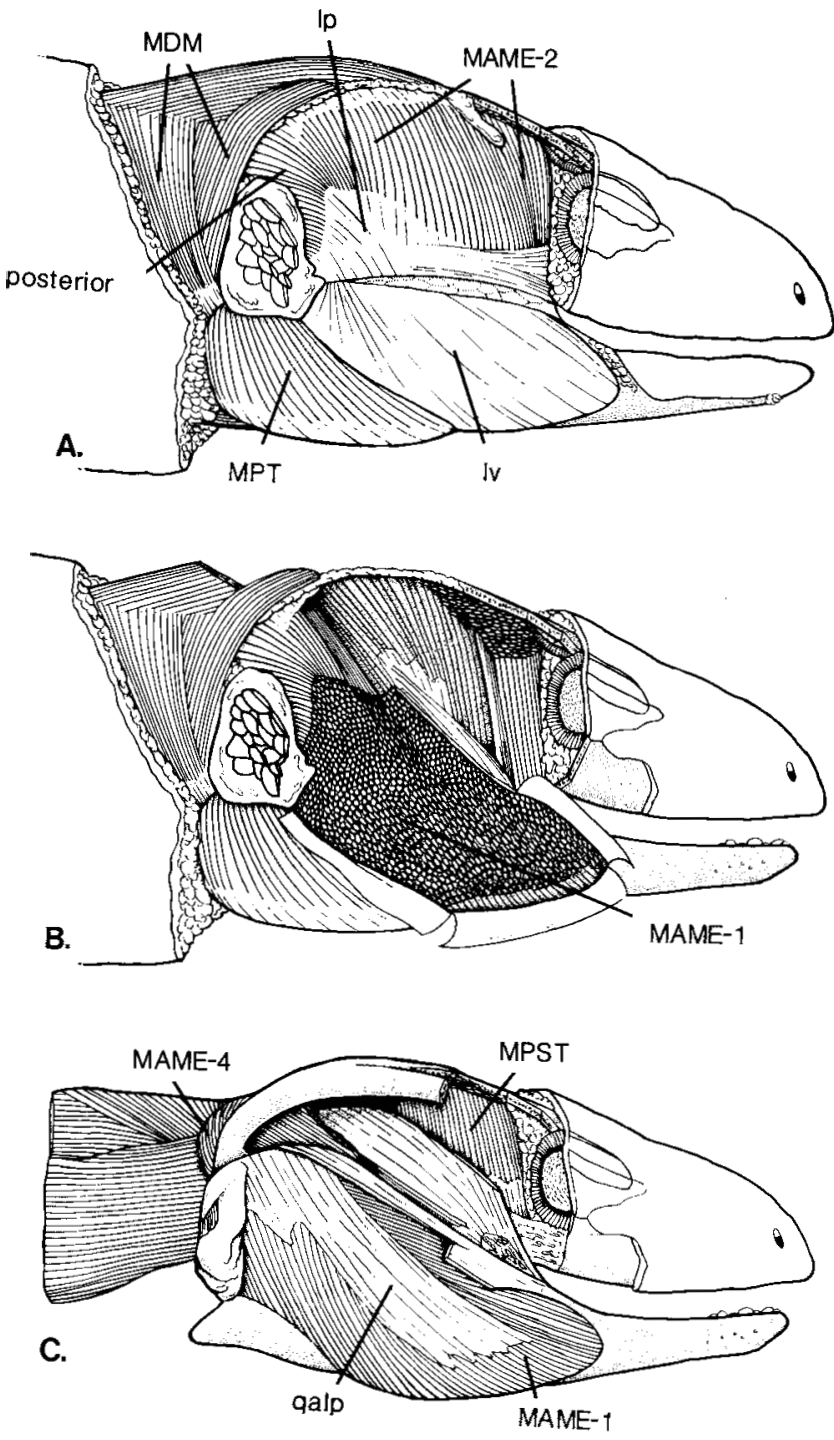

D.

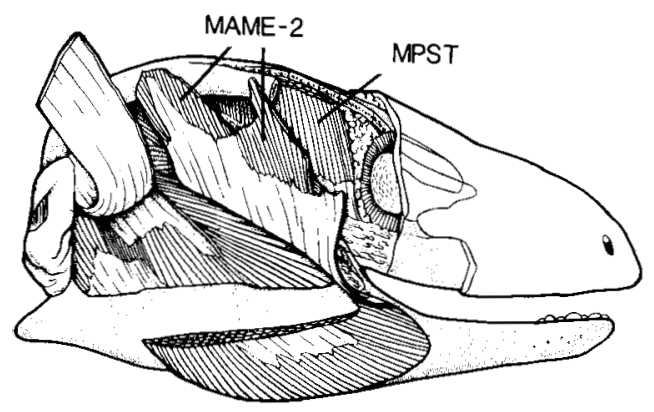

Fig. 1. Trachydosaurus rugosus. Lateral views of the head to show the musculature. A-D show increasingly deep dissections. Note the shift of muscle architecture, fiber direction, and fiber length as the layers are separated. Fiber placement is to be interpreted in terms of the relation of the insertion site to the position of the jaw articulation. MAME-1, $-2,-3,4, \mathrm{M}$. adductor mandibulae externus, parts 1-4; MPT, M. pterygoideus; MPST, M. pseudotemporalis; Ip, Iv, aponeurosis posterior and ventral. (Figures modified after Wineski and Gans, 1984.) 

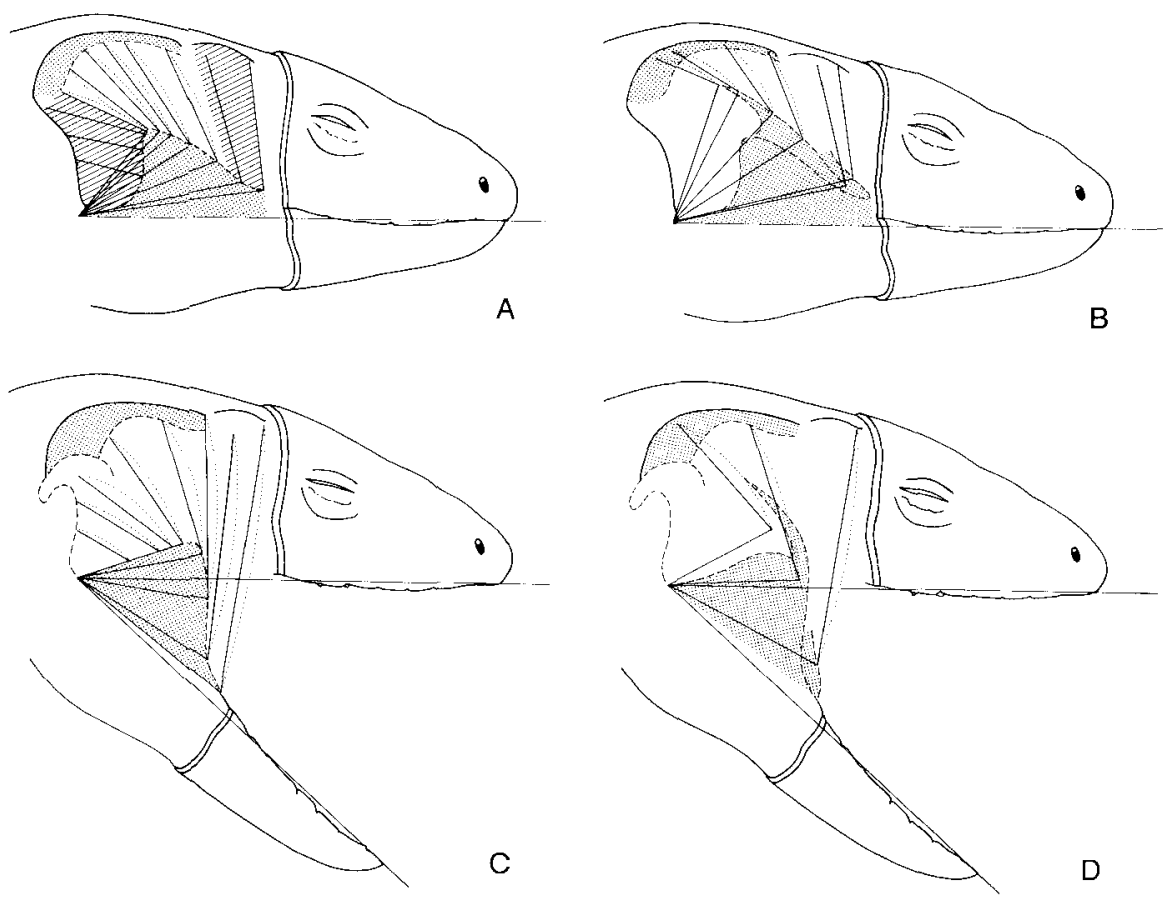

Fig. 2. Trachydosaurus rugosus. Lateral sketches of the main adductor mass to show changes in fiber orientation and length from the open to the closed position of the mandible. A, C, superficial; B, D, deep; A, B, closed;

the angle of the mouth and glued to the labial skin.

\section{MAME-2 middle}

The needle was passed ventrally from the dorsal portion of the postorbital region. In one experiment three electrodes were placed adjacent and in series.

\section{MAME-2 posterior}

The needle was passed from the posterodorsal portion of the postorbital region and in a posteroventral direction toward the dorsal aspect of the quadrate.

\section{MAME-3 posterior}

The needle was passed in a ventral and slightly medial direction, medial to the supratemporal process.

\section{MAME-4 posterior}

The needle was passed from the posterolateral aspect of the head, through the posttemporal fossa, until the dorsolateral aspect of the prootic was contacted.

C, D, open. The portions of the MAME-2, referred to, respectively, as anterior, medial, and posterior, are indicated by differential hatching.

\section{Pseudotemporalis, superficial (MPSTs)}

The needle was inserted from the inside of the mouth to pass in a dorsomedial direction directly into the anterior portion of the muscle. The wires were led off through the angle of the mouth and glued to the labial skin.

\section{Pterygoideus, superficial (MPTs)}

The needle was passed from the lateral face of the posterior aspect of the mandible until it reached the retroarticular process. The wires were led off through the angle of the mouth and glued to the labial skin.

\section{Pterygoideus, profundus ( $M P T_{\mathrm{p}}$ )}

The needle was passed posterolaterally through the external aponeurosis via the open mouth. The wires were led off through the angle of the mouth and then glued to the skin.

\section{Depressor mandibulae, anterior (MDM)}

The needle was passed through a point just back of the posterior margin of the external auditory meatus. 


\section{Cervical muscles}

The needle was passed from behind, to penetrate the integument in the nuchal region, so that the tip rested in the band of muscle between the adductors.

\section{Deep cervical muscles (rectus capitis group)}

The needle was passed through the pharynx and entered the muscles posterior to and between the bulging pterygoid musculature in the roof of the mouth.

Several heads were dissected and the muscles were dissolved in $20 \%$ nitric acid until their muscle fibers could be separated with glass needles (Williams and Goldspink, 1971). Fiber lengths were measured for each of the tendinous compartments of the several muscles. The action lines of the muscles were checked by comparing them for the specimens preserved with the mouth closed and two preserved with the mouth open.

A separate set of experiments determined the change of force with jaw angle in vivo. Anesthetized animals were positioned on a horizontal platform with the mandible horizontal and the head clamped at various angles of elevation. A vertical stainless-steel wire connected the mandible to a fixed-force transducer, the output of which was passed through one channel of a Honeywell 117 operational amplifier, stored on tape, and displayed on a Brush 8100 chart recorder simultaneously with the signal from the stimulator. A Grass S-44 stimulator generated continuous signal trains and a toggle switch allowed the application of a selected number of these pulses to the animal. Stimulation (single or trains of pulses) was via stainless-steel (insect pin) electrodes inserted through the dermal armor for $1 \mathrm{~cm}$ into the front and back of the adductor musculature. Durations ( 5 msec) were kept low to avoid damaging or unduly fatiguing the muscles. Trials with higher values increased the force maxima, but not the patterns observed. All measurements were isometric. The degree of mouth opening was varied to determine the relation between jaw angle and adductor force.

\section{RESULTS \\ Behavior}

Shinglebacks are clearly omnivores, feeding on a variety of foods that vary in consistency and escape behavior. Here we characterize two extreme patterns of ingestion and crushing: tomato segments and snails of varying sizes (and crushing strength).

Whenever food is introduced into the cages, shinglebacks flick their tongue. As soon as chemical cues are perceived (immediately, if the tongue of a hungry animal is allowed to touch food objects or an instrument smeared with juice), conditioned animals move toward the food. They are easily trained to take food objects from forceps. Red objects and moving animals appear attractive and the lizards quickly learn to recognize the sound of snails being dropped into the cage. Their rate of movement and the distance shinglebacks traverse upon emerging from a shelter reflect recent feeding history. If they have not obtained fruit for a week or more, they are motivated by tomatoes or plums. After a week of feeding exclusively on fruit, they walk more rapidly toward a newly introduced snail or cricket.

Once a prospective food item has been contacted by the tongue, a bite generally follows (Fig. 3A). The head of these lizards is wide, the body stout, and the limbs short; in the resting position, the mandible lies in full contact with the substrate. From this position, the mouth could only be opened by raising the upper jaw; the skull must then be elevated on the occiput and the cervical column curved. Generally, the head is lifted by extension of the forearms prior to mandibular depression per se; this movement tends to proceed with forward rotation of the trunk over these props. If the food object is as large as the depth of its head, the lizard may bite straight forward. Shinglebacks can also bite downward at an angle of close to $60^{\circ}$ (from the horizontal) by raising the body on the forelimbs and rotating the snout ventrally. More commonly they rotate the head up to $90^{\circ}$ on the long axis until the mouth can be closed horizontally with both upper and lower jaws parallel to the ground; this permits Trachydosaurus to pick up very small food objects.

During the threat display, a hyoid linkage may lift the leaf-shaped lingual pad of the flexible and highly protrusible tongue; the tongue may then be tilted over and past the mandibular symphysis, thus being completely everted during the very wide gape. During food pick-up, the tongue is protracted until it contacts the food; the tip is then depressed, so that the ruffled, initially dorsal surface rotates forward. A highly viscous mucus coats the dorsal lingual surface and makes it distinctly more adhesive than the 

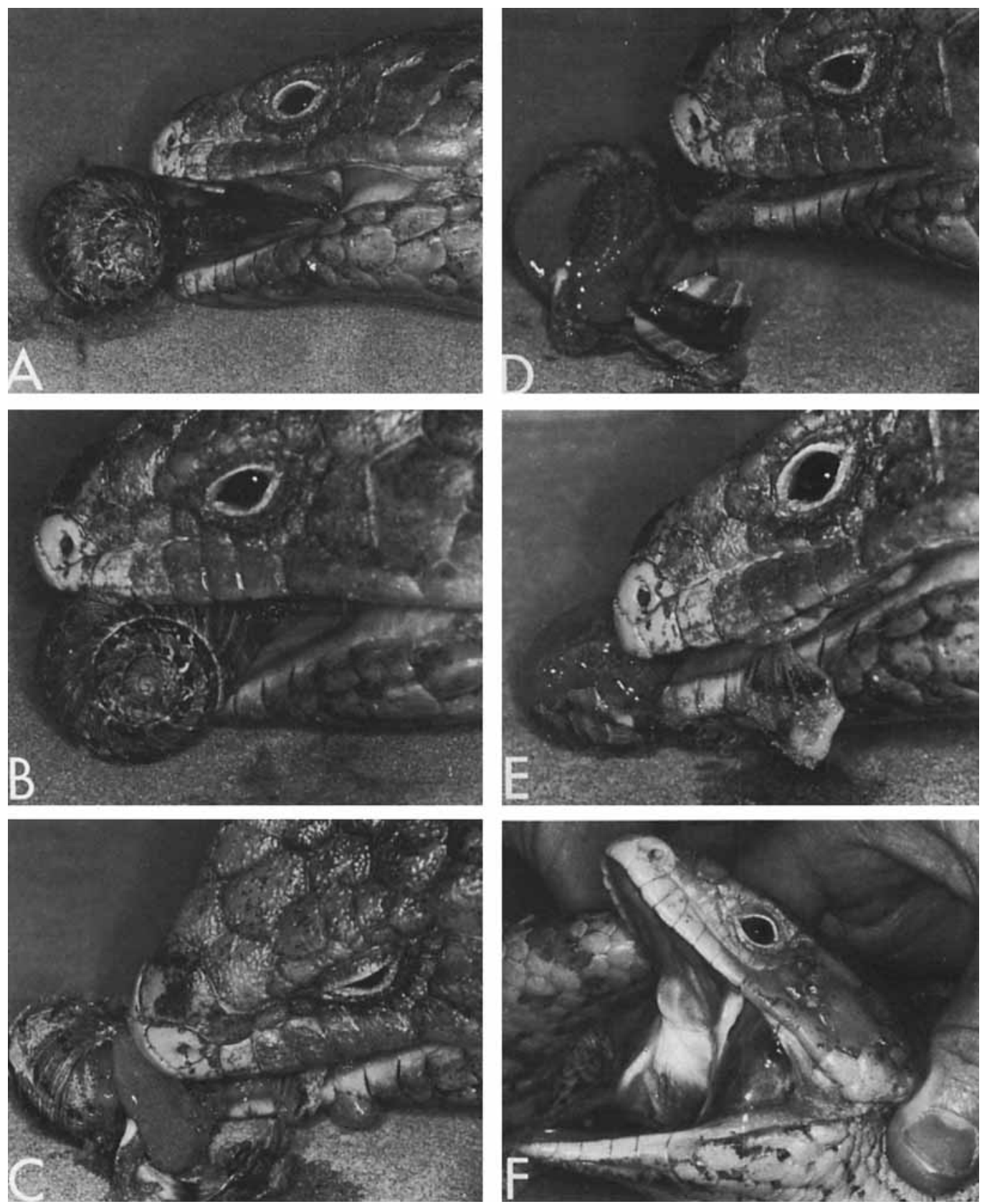

Fig. 3. Trachydosaurus rugosus. Montage of photos taken during a snail capture, crushing, and reduction sequence, as well as the defensive gape. A. Horizontal bite at prey showing the rotation of the head on the long axis of the trunk and the contact of tongue with prey just prior to the retraction that will pull the snail between the tooth rows. B. Prey between the tooth rows

during a crushing bite. C. Snail with broken shell discharged after the initial bites; fragments are being separated. D. Secondary bite at the soft parts of the snail. E. Lateral wiping movement of the tongue drives a shell fragment from the side of the mouth. F. Defensive bite of a lizard while being held (the tongue display is rarely used if the the animal is being restrained). 
ventral one; capillary effects let the former stick effectively to hard, smooth, and dry materials (i.e., tomato skin, snail shell) (Smith, 1984). The low-viscosity fluid coating of some soft and wet foods (cut surface of fruit) permits separation as the fluid film is loaded in tension.

The first biting movement may or may not involve use of the tongue (Fig. 3A). Generally, the tips of the jaws approach and tend to bite at the food object. During the second bite (also if the food object has been released or was missed) the mouth again gapes widely. As soon as the tips separate enough to clear the food, the dorsal surface of the tongue is pushed into contact, generally achieving adhesion on hard materials; lingual retraction then accelerates the food object into a position at which the tooth rows can close on it (Fig. 3B). The bluntly conical teeth can crush and hold, but are relatively ineffective for cutting. Fruit and prey may be broken by biting and shaking or scraping off the protruding portions against the ground.

The "open-shift prey with tongue-close" pattern continues through the masticatory sequence. The tongue can apparently be slipped beneath the prey, even with the mouth closed; its lifting action during the opening phase would pull the food object off the mandibular tooth rows, whereas the simultaneous effect of gravity separates it from the maxillary teeth. One reason that the jaws separate among masticatory cycles is that this promotes disengagement of jaws and food and reduces the force that the tongue must exert in order to move the prey.

Simple static-loading tests indicate that the force that must be applied to break the shells of snails differs by more than a factor of five with the orientation of force application. There was no indication that shinglebacks make decisions about the several initial positions. The orientation of the starting bite is clearly random. However, the tongue rotates the snail each time the mouth opens so that each successive closure tends to impact at a different position.

Snails may be crushed during the first, second, and even third bites of a snail-feeding sequence; the crushing bite is as much as $\mathbf{1 . 5}$ to two times longer than the bites that follow (Table 1). If the snail is large, the tip of the lower jaw is usually held firmly against the substrate and the upper jaw is moved downward against the fixed prey. Should a large food object be picked up with a lateral bite,
TABLE 1. Duration (msec) of open/close cycles in sequences of snail crushing by Trachydosaurus ${ }^{1}$

\begin{tabular}{ccc}
\hline Bite No. & $\begin{array}{c}\text { Duration of adductor } \\
\text { activity } \\
\text { (mean } \pm \text { S.D.) }\end{array}$ & $\begin{array}{c}\text { Length of cycle } \\
\text { (mean } \pm \text { S.D.) }\end{array}$ \\
1 & $840 \pm 340$ & $1,260 \pm 440$ \\
2 & $440 \pm 290^{*}$ & $890 \pm 340^{*}$ \\
7 & $370 \pm 230$ & $780 \pm 320$ \\
8 & $380 \pm 230$ & $780 \pm 260$ \\
14 & $430 \pm 170$ & $840 \pm 270^{*}$ \\
15 & $320 \pm 180$ & $760 \pm 340$ \\
\hline
\end{tabular}

${ }^{1}$ Bite 1 is set at the initial crushing bite. All subsequent bites are significantly different from the initial bite $\left(P<.001\right.$ or ${ }^{*} P$ $<.05, \mathrm{~N}=17$ ).

the head is first lifted returning it to horizontal; the mandibular symphysis is secondarily placed into contact with the ground. During the manipulative and reduction bites that follow crushing, the head is lifted and held relatively stationary, and only the lower jaw moves. Smaller snails may be crushed during first bites with the head lifted.

On superficial examination, the skull appears to show no kinesis and the lips show minimal flexibility. No streptostyly could be documented in cinefluoroscopy (although this technique would likely miss displacements of $1 \mathrm{~mm}$ or less). However, because the jaw joint does not lie on the posterior continuation of the lips past the angulus oris, the mandible and the edge of the ossified labial integument do not move in parallel. As they are closely connected anteriorly, there has to be some relative displacement posteriorly.

Shinglebacks often bite food objects asymmetrically, transmitting most of the force via the mandible of one side only. Whenever a single ramus impacts on a hard object near the symphysis, one may see the unloaded ramus rise away from the ossified integument in a very asymmetrical closure. Also, bites into a hard object deform the supralabials very locally, generally along a horizontal bending line midway up their height; each of these scales is locally anchored to the underlying bone. In contrast, the row of infralabials deflects as a unit, exerting general pressure on large objects held in the mouth and indicating that the infralabials are more loosely connected to the underlying bones.

Subsequent crushing and ingestion of snails proceed by trial and error. Each snail is bitten repeatedly, being shifted between the tooth rows and thoroughly mashed. In- 


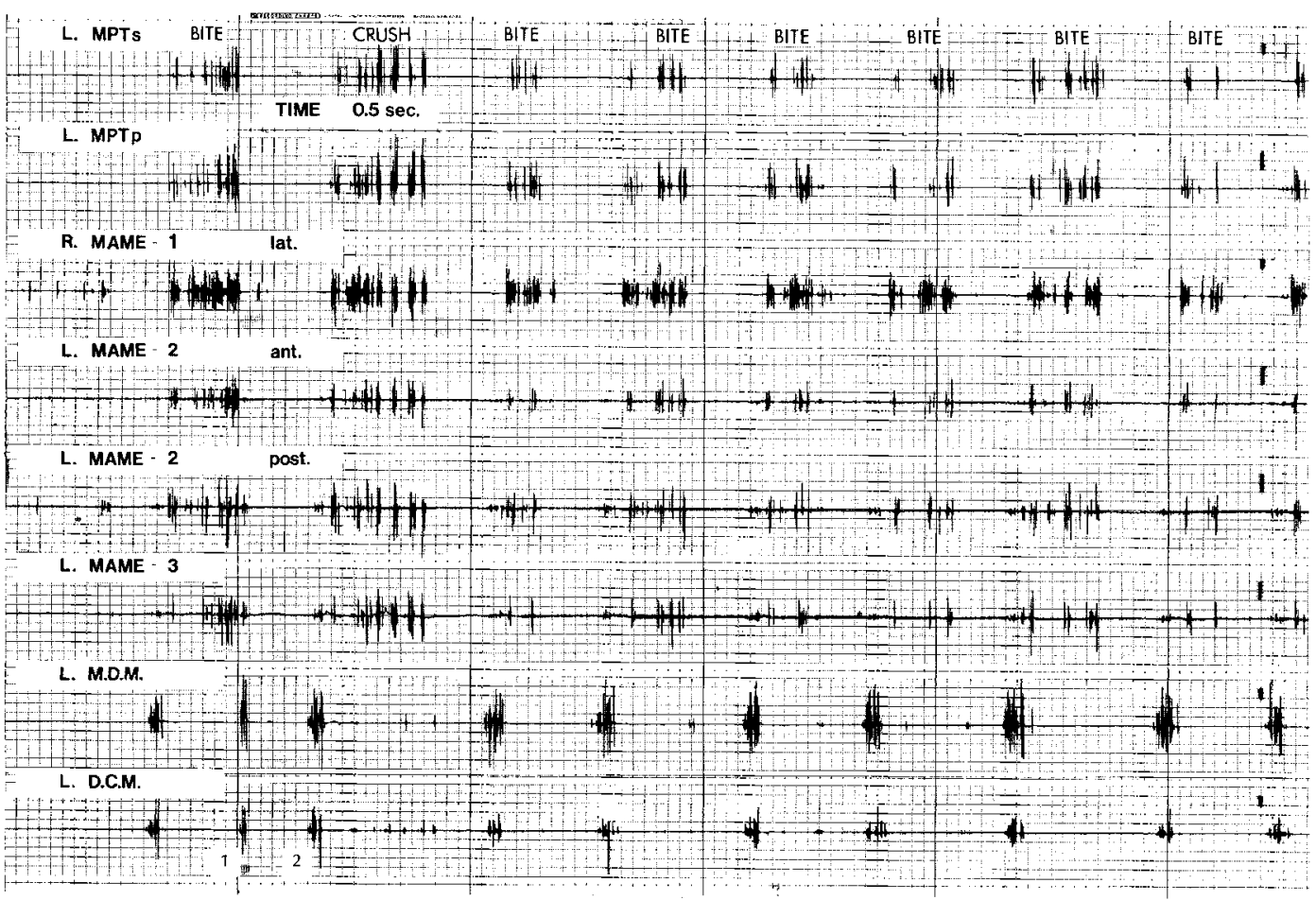

Fig. 4. Trachydosaurus rugosus. Electromyograms of six adductors and two depressor muscles during the crush and the initial bites of a snail mastication sequence. The snail slipped from between the jaws during the first bite and the opening activity (1) that follows is repeated (2) without an intervening closing movement. The next bite shown represents the crush of the snail as indicated by the synchronized pulsatile activation of the adductors. The time markers indicate $0.5 \mathrm{sec}$ and the vertical bars

termittent protrusion of the tongue scrapes bits of shell from the mouth. At least once in most crushing sequences, shinglebacks, presumably accidentally, will scrape out the soft parts of the snail (Fig. 3C-E). Obvious searching movements follow; one object after another is picked up until the soft parts are located and reingested. The search appears to be steered by tactile and olfactory cues, mainly the latter, as shinglebacks will tongue and then pick up and crush empty pieces of shell, but never pebbles. Visual cues do not seem to be used at this time; the soft parts of the snail are ignored even if they move just to one side of the jaws. Pick-up, crush, scrapeout sequences follow each other until the closing movements only encounter soft tissues; swallowing then follows. on the right $0.1 \mathrm{mV}$. L-MPTs, left superficial pterygoideus; L-MPTp, left deep pterygoideus; RMAME-1, right adductor mandibulae externus 1, lateral portion; LMAME-2, left adductor mandibulae externus-2, anterior portion; LMAME-2, left adductor mandibulae externus-2, posterior portion; LMAME-3, left adductor mandibulae externus-3; LMDM, left depressor mandibulae; LDCM, left dorsal cervical muscles.

The capture pattern appears to be astonishingly clumsy. Two to six bites are often necessary to grasp stationary food objects effectively or to let the adhesive effect of the tongue pull an object between the tooth rows. However, the lizards are highly persistent. If food objects roll or insects move away, the shinglebacks steadily follow. Handling of sliced fruit indicates a most limited capacity to respond to unusual topologies. A scan of some hundreds of feeding sessions provided no evidence that the animals learn to deal with spatial characteristics of the prey nor that they can recognize instability of objects. Attempts at biting or the touch of the protruding tongue often displace the snail or tomato. However, the lizards offer minimal correction of the angle of the next bite; they 
repeat the movement until the food object is "accidentally" maneuvered into a position from which it does not roll away.

\section{Motor activity}

The mouth of Trachydosaurus is normally closed. A biting cycle is here defined as starting at the instant the mouth begins to open, the head being elevated and the lower jaw then being depressed. The lower jaw drops and the tongue may be partially protruded and then retracted, sticking to and manipulating the food object. Thereafter the jaws close, impacting upon the food object and crushing it. Depending upon the nature of the food, some 40-100 closing cycles precede swallowing, which may be followed by a series of cleaning cycles during which the tongue sweeps out the mouth. Muscle activity during the start of a reduction sequence is shown in Figure 4.

Approximately $100 \mathrm{msec}$ prior to opening of the mouth, the Mm. cervicales and depressor mandibulae become active for approximately $175 \mathrm{msec}$. Roughly $150 \mathrm{msec}$ later, the posteriormost portions of the adductor mandibulae externus (posterior MAME-1 and MAME-2, MAME-3 and MAME-4), part of the adductor complex, also show an initial burst of low activity. This activity occurs during opening of the mouth and ends simultaneously with the cessation of activity in the depressor musculature. Activity in all of the monitored muscles (both depressors and adductors) typically ends abruptly and there follows a silent period of variable duration (40-110 msec) during which the mouth is open and food continues to be manipulated by the tongue.

Activity in the adductor complex ends the pause and within 30-120 msec the mouth begins to close. Most of the adductor muscles, including the $\mathrm{Mm}$. adductores mandibulae externus and pseudotemporales, become active more or less simultaneously. Only the M. pterygoideus become active slightly (0$150 \mathrm{msec}$ ) later than the other adductors.

The duration of activity in the adductor complex varies dramatically depending on the physical properties of the material being reduced. Adductor activity can last as long as $0.8-0.9$ seconds during the initial crushing of snail shells, or be as brief as $0.2 \mathrm{sec}-$ onds whenever the animal is chewing on soft materials, such as snail bodies or tomatoes. The duration of the adductor phase of the bite cycle also changes in response to the

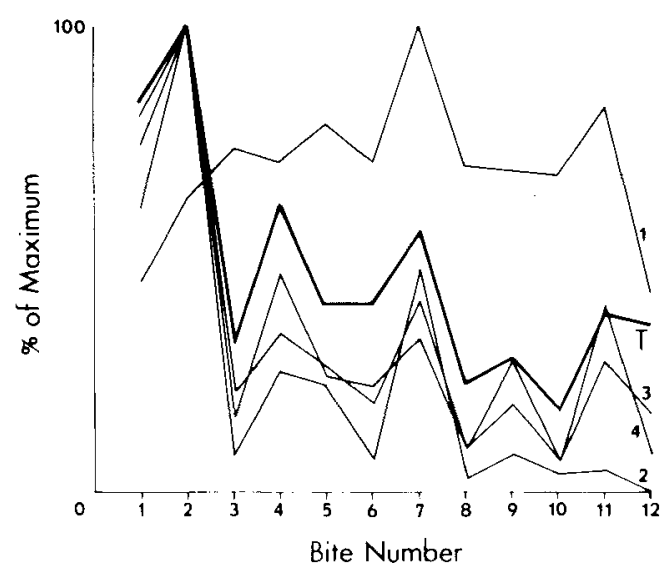

Fig. 5. Trachydosaurus rugosus. Typical graph giving length and magnitude of muscular activity as a function of bite number in a series. Aggregate values of duration of muscular activity (heavy solid line labeled T) and EMG activity expressed as number of spikes times mean amplitude (thin lines) for the depressor muscle and three adductors. 1, depressor mandibulae; 2, right lateral MAME-1; 3, left lateral MAME-1; 4, left pterygoideus. Note the difference in variation in adductor and depressor activity among the bites of the series. Also note that the duration directly parallels the increased product displayed by the adductors during the crush, thus indicating that this increase reflects longer rather than more intense muscular activity.

texture of the material being reduced; it is near 55\% in crushing and $20 \%$ in chewing.

Figure 5 shows the product of spike number per sampling interval and the mean amplitude for that interval versus the bite number within a reduction sequence. The number of spikes and mean spike amplitude per interval generally do not change significantly between the initial crushing bite and subsequent chewing bites. However, the graph indicates that the aggregate product of the initial and crushing bite differs from that of the later ones, an effect that is entirely due to the length of time occupied by the crushing bite. Thus the product (spike number times mean amplitude) for a bite is directly correlated with the length of closure $(r=.94)$.

Whereas the activity of the adductor musculature during crushing does not show significant differences in terms of amplitude and spike frequency from that during biting, the former activity is characterized by a sequence of synchronized and repeated pulsations (Fig. 4). The individual bursts last 40 
TABLE 2. Lengths of fibers in major jaw muscles $(\mathrm{mm})(N=100)$

\begin{tabular}{lcr}
\hline & Mean \pm S.D. & Range \\
\hline M. adductor mandibulae externus, & & \\
part 1 (MAME 1) & & $8.0-9.9$ \\
Lateral, anterior & $8.76 \pm 0.54$ & $6.2-8.3$ \\
Lateral, posterior & $7.07 \pm 0.45$ & \\
Deep, lateral & $11.28 \pm 0.74$ & $9.9-12.6$ \\
Deep, intermediate & $10.49 \pm 0.90$ & $9.2-12.2$ \\
Deep, medial & & \\
M. adductor mandibulae externus, & & $15.2-17.0$ \\
part 2 (MAME 2) & $16.02 \pm 0.47$ & $12.3-14.2$ \\
Anteriormost & $13.04 \pm 0.57$ & $9.0-10.5$ \\
Middle & $9.87 \pm 0.39$ & $8.5-10.6$ \\
Posteriormost & & $9.63 \pm 0.68$ \\
M. adductor mandibulae externus & & $9.0-10.5$ \\
Part 3 (MAME 3) & $9.77-0.34$ & $6.2-7.2$ \\
M. adductor mandibulae externus & $6.49 \pm 0.20$ & $12.0-13.6$ \\
Part 4 (MAME 4) & $12.86 \pm 0.37$ & $9.7-12.0$ \\
Posttemporal fossa & $10.73 \pm 0.78$ & $9.8-11.0$ \\
M. pseudotemporalis superficialis (MPSTs) & & $6.8-8.2$ \\
M. pseudotemporalis profundus (MPST) & $10.45 \pm 0.36$ & \\
M. pterygoideus superficialis (MPTs) & $7.44 \pm 0.38$ & $6.0-7.5$ \\
Lateral & & $4.2-5.8$ \\
Medial & $6.87 \pm 0.41$ & $5.9-7.0$ \\
M. pterygoideus profundus (MPTp) & $5.09 \pm 0.41$ & $4.9-6.2$ \\
Medial & $6.40 \pm 0.32$ & $22.1-25.0$ \\
Lateral & $5.52 \pm 0.42$ & $18.2-21.0$ \\
M. protractor pterygoidei (MPPt) & & $15.4-18.0$ \\
M. levator pterygoidei (MLPT) & $23.85 \pm 0.88$ & \\
M. depressor mandibulae (MDM) & $19.39 \pm 0.91$ & $16.77 \pm 0.87$ \\
Anteriormost & & \\
Post. part of anterior & & \\
Posteriormost & & \\
\hline
\end{tabular}

75 msec and occur with a frequency of 6.8 to eight per second. As many as nine of these short bursts may occur during a crushing bite. This pulsatile activity is most characteristic of initial crushing bites on snail shells but may be observed during some subsequent bites.

The different components of the adductor complex generally become silent synchronously. Cessation of activity in the adductors is followed by a relatively long and variable silent period (350-700 msec), during which the mouth remains closed.

The dorsal and deep cervical muscles fire whenever the head is lifted or depressed; their action is asymmetric whenever the head is shifted from side to side. The deep (subvertebral) cervical muscles are active throughout a reduction series; they then fire just before and after the action of the depressors. However, they also fire strongly during the initial crushing bites, in which the mandible contacts the ground. During such a bite, the subvertebral muscles fire in synchrony with the adductors; during a reduction series they do not and the pattern becomes asynchronous as soon as the mandible is lifted out of contact with the ground.

\section{Fiber length correlation}

To the earlier report of the morphology (Wineski and Gans, 1984) is here added a table of fiber lengths for both sides of one specimen with the mouth closed (Table 2); the relative lengths of the muscle fibers measured on three additional heads did not show significant differences. However, absolute measurements differed and elongation with opening produced changes between 10 and $100 \%$ of fiber length with the mouth closed (Fig. 2).

The length and insertion angle of the fibers of the MAME-2 were mapped to check their correlation with fiber placement (Fig. 2). The best correlations were obtained with the mouth closed. The anterior and medial fibers of the MAME-2 (constituting most of the adductor mass) then inserted at angles between $87^{\circ}$ and $102^{\circ}$ to the line connecting the site of insertion with the center of rotation (at the joint). The range of angles shifted to $60^{\circ}$ and 
$82^{\circ}$ whenever the mouth was open. However, the angles of these fibers relative to the line between fiber and long axis of the mandibular ramus ranged far more widely (from $38^{\circ}$ to $83^{\circ}$ ). This suggests that the former angle is more likely to be significant to the animal than is the latter. For most of the muscle, the fiber length correlated directly with the absolute distance between fiber insertion and instant center of rotation. The insertion angle of the posteriormost fibers, inserting on the posterior edge of the aponeurosis-I, differs from that of the main adductor mass (Fig. 2). These fibers may serve mainly to tense and maintain the position of the aponeurosis.

\section{Stimulation experiments}

The adductor mass was stimulated by pulses at frequencies between 5 and $12 \mathrm{~Hz}$, a range including the pulsatile frequencies observed during crushing. This stimulation pattern generated a staircase summation of force, adding approximately $50 \%$ of the initial value for the second pulse, $50 \%$ of this for the third, and so on; the increments depend upon the initial stretch of the muscle, the stimulus voltage, and the rate. By the time of the fourth and fifth pulses, the force approaches the asymptote. In one experiment on one specimen, a small snail was placed between the jaws and the stimulus voltage was increased without achieving fracture of the snail.

Figure 6 shows the twitch values plotted versus the angular opening of the mouth. Whereas the actual tension values differed for each animal, the curves generated for each animal showed peak moments at or near maximum opening as well as a substantial ( $>50 \%$ ) decline of moment near the close.

\section{DISCUSSION \\ Technical aspects}

The results of the field experiments showed patterns that did not differ significantly from the recordings made in the laboratory. Frequency response and similar aspects were acceptable as indicated by the equivalent nature of the digitized results. The main difficulty with the four-channel tape system was the problem of making decisions about the reality of synchronization among the activities of multiple muscles. This problem was exacerbated by the almost simultaneous onset of activity in many muscle groups. The need to repeat experiments in order to record

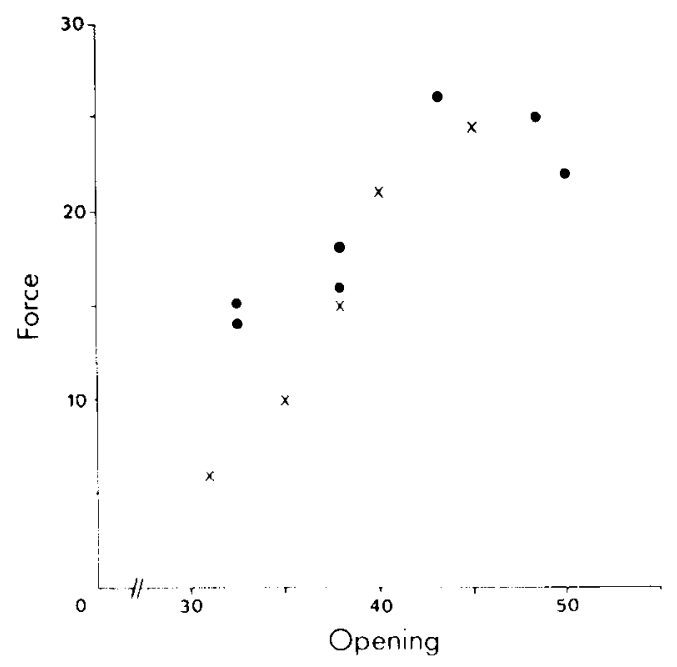

Fig. 6. Trachydosaurus rugosus. Results of experiment in which lateral adductor mass of an anesthetized animal was stimulated submaximally. Plot of moment (due to single twitch stimulation) measured at the mandibular symphysis by a strain gauge as a function of various degrees of opening expressed as the linear distance (in $\mathrm{mm}$ ) between the mandibular tips (corresponds to angular opening in degrees, $\pm 3^{\circ}$ ). Force scale arbitrary. Two trials on a single animal shown. Solid circles, run 1 ; crosses, run 2 .

(and then to compare) the records of particular pairs of muscles from a single (four-channel) record complicated attempts to estimate the influence of slightly different food objects, angles of attack, states of motivation, and other variables. As this was the only significant limitation of the field approach, in the future it could be dealt with by using tape recorders with at least seven or more recording channels or by synchronizing two four-channel systems.

Recordings from compartments of large, complex muscles require confirmation that the signals were indeed generated from the segment in question. In the present case, the heavy integumentary osteoderms made the insertion of barrier or patch electrodes impractical. Consequently, electrode placement was checked by dissection and the likelihood of bulk conduction was checked by examining the level of coincidence of spike pattern among records from adjacent electrodes (muscles).

Recordings from the MAME-2, MAME-3, and MAME-4 indicate low-level bursts of fir- 
ing during the opening phase, coincident with activity in the depressor and cervical levator muscles. It is possible, but unlikely, that these represent cross-talk, due to bulk conduction from the mouth-opening and headlifting systems. However, numerous records show noncoincidence of the spike pattern and asynchrony between the signals in adjacent sites. Although the firing intervals are coincident, the records of successive bites show distinct asynchronies. Thus, the similarity appears to be due to coactivation.

The electrodes and their placement were insufficiently standardized and the biting pattern too irregular to let us compare activation of left versus right sides. The sides do show slight and varying asymmetries of action, presumably due to asymmetrical placement of the food.

The initial expectation that the magnitude of masticatory force required for different food types might be derivable from the EMGs (as established for mammalian studies, Gorniak and Gans, 1980) proved to be simplistic, at least for the ingestion of snails, as could have been extrapolated from the behavioral data. Unlike the patterns described for mammals, in which the force remains substantial throughout a reduction sequence, the shinglebacks exerted most force during one of the first bites of a snail-feeding sequence. This peak force was clearly coincident with fracture of the shell, rather than with the further crushing of the soft parts. However, subsequent movement cycles differ as the food continues to be repositioned, as shell remnants are cleared out of the mouth and as remnants of the shell or soft portions of the snail are crushed or compressed. The continuous between-bite rotation of the bolus, whether snail or tomato, and the intraseries uncertainty about kind and texture of the portion likely to be met by the teeth are responsible for the oscillations in the EMG values of successive bites and make simple correlations impossible.

\section{Implications of fiber arrangements}

\section{Physiology and behavior}

The architecture of a masticatory system may have been determined variously by phy. logeny and function-most likely by interaction of the two. Ultimate resolution of the constraints requires a comparative approach (cf. Gans, 1985). However, the structure/function association can be analyzed for a specific case. Whereas the observed function implies utilization, its possible role can only be established by an ecological analysis, which is beyond the frame of the present project. We here attempt to establish the extent to which the architecture of the system permits various functions, remembering that the phylogenetic component may have involved selection for different roles at earlier stages of phylogeny.

Whereas the musculature of Trachydosaurus does not show temporal subdivision, the other aspects indicate that increased force is a major determinant in the organization of the mandibular system. First of all, the jaw opening-force analysis indicates that the moment is maximal for opening of more than $30^{\circ}$. (This contrasts with the values for man in which maximum adductor force is reached well before the mandible is halfway depressed, Mackenna and Turker, 1983.) That force is important derives from observations of the movement pattern and the attitude used when the animals crush very large, hard objects. At this time, the mandible is held against the ground and the mandibular musculature pulsed. Action of the ventral neck musculature further depresses the head and acts synergistically to enhance the crushing force. All muscles involved are activated in a pulsatile pattern. This confirms the observation that unilateral stimulus pulses are in. sufficient for reduction of large snails.

Trachydosaurus have a rather substantial gape and perhaps ten times more muscle mass devoted to closing than to opening the mouth. (Statements about this relation will be complicated as some of the neck muscles contribute to opening and closing the jaws. Also we have not studied the activity of the hyoid muscles.) The closers are in a mechanically much more advantageous position than is the depressor mandibulae. Cinefluoroscopy indicates that shinglebacks lack substantial cranial kinesis; for that matter, they lack any obvious capacity for streptostyly or propalineal movement of the mandible.

In contrast to the simple mandibular movements it produces, the adductor musculature is remarkably complex. At least 11 major subdivisions are easily identified (Wineski and Gans, 1984; Table 2, Fig. 1) and some of these show major differences in fiber length and direction. Complicated, scrolled sheets of connective tissue subdivide the muscle-filled space, and adjacent compartment sections show enormous differences in proportions and in the direction of their fibers. The absence 
of temporal differences in activation and the lack of kinesis make this an ideal case for testing the functional bases of such fiber arrangements. Consequently, we here state some basic relations of muscle architecture and then test them against the arrangement observed.

\section{Theory of fiber arrangements}

The force generated by a muscle differs with the length at which the muscle is activated-in other words, with the position of the component sarcomeres on the length-tension curve (Gordon et al., 1966). We assume, for purposes of the initial analysis and within the limits established by different fiber types, that not only will all sarcomeres of parallel fibered and regularly pinnate muscles have equivalent properties, but that these sarcomeres will at any one time occupy equivalent positions on the length-tension curves. This assumption needs testing for different muscles acting on the jaws as well as for all of the fascicles of any single muscle (Herring et al., 1984). However, equivalence is initially assumed, as is the static behavior of the system.

Complexity is reflected in fibers arranged along lines lying at angles differing from those of the resultant forces transmitted by the tendon of the whole muscle and also substantial differences in length among the fibers of a muscle (Gans, 1982; Gans and Bock, 1965). Several reasons have been or may be adduced to explain muscles that show complexly arranged fibers between the surfaces of origin and insertion. These are 1) subdivision of labor, 2) potential shift of insertion site in different directions relative to the site of origin, 3) adjustment of fiber length (and number) to maintain advantageous force-excursion relations, and 4) packaging of the muscle mass to fit available configuration.

1) The first and perhaps most obvious basis for complexity is that the several muscles, muscle fascicles, and motor units may be arranged at different angles and may be activated at different times in the cycle(s) of movement. Subdivision of labor could allow their differential recruitment during the opening or closing sequence of the mandible, perhaps close to their mechanically most advantageous position.

Mechanical advantage changes with rotation of the jaws. The angle of muscular insertion on a rotating element, such as the mandible, tends to change with mandibular rotation. Muscles that insert normally whenever the jaw is open then generate their greatest moment and this decreases as the mouth closes (Fig. 7). The moment arm of muscles that insert normally whenever the jaws are closed will drop as the mouth opens. The moment arm will be affected not only by the distance between the site of insertion and the jaw joint, but also by the elevation (or depression) of the insertion site above or below the line that connects the joint and tooth cusps (Fig. 10A).

The plateau or resting length (at which the individual sarcomeres generate maximal isometric force) may be placed at any degree of opening by changing the number of sarcomeres in series; this may affect the fraction of the distance between origin and insertion that is occupied by muscle rather than tendon (it may also affect the flatness of the muscle's length-tension curve). If sarcomeres are set so that their resting length coincides with that of greatest instantaneous moment arm, the moment values will peak there (Fig. 7). If their resting length coincides with a lower moment arm, the moment may be relatively constant throughout the range of jaw positions (as the curves of force and moment cross within the working range).

Distinct dentitions and different food types may establish advantages for distinct times and courses of crushing-force application. Thus, maximum moment might be needed whenever the jaws of a crusher are widely separated; for a ruminator, it might be advantageous to exert maximal force at a position close to occlusion, as it is for man (Mackenna and Turker, 1983). It is easy to see that different muscles could produce their maximal moment at different degrees of opening. Consequently, there exists the potential that they might be activated at different times-for instance, near mechanical optimum. This might represent one set of reasons for functional subdivision, leading to complexity and differential activation.

2) The second explanation would be that complexity allows lateral or propalineal shifts of the insertion site by the action of muscles with crossing action lines, as seen in mammalian mastication. This would presumably require that the mechanical parts indeed be movable laterally. Beyond this, one would have to demonstrate that laterally displaced components of the musculature show differential activity. A variant of this would be placement of pairs of muscles so that they 


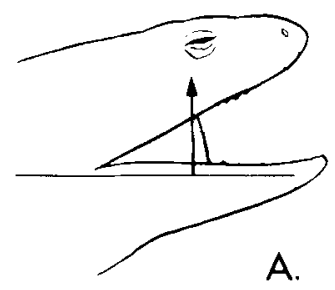

A.

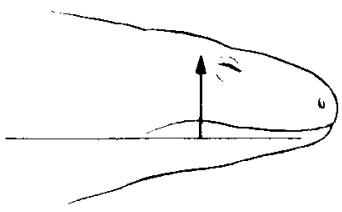

B.
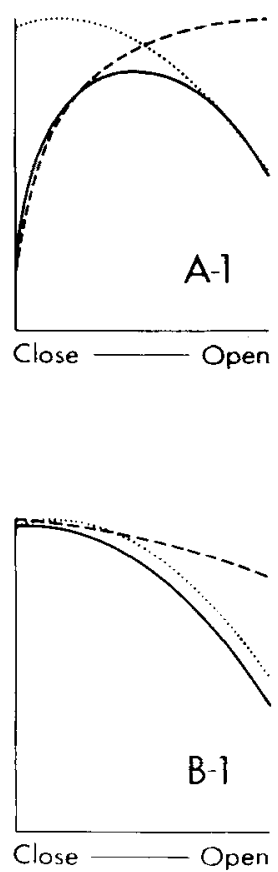
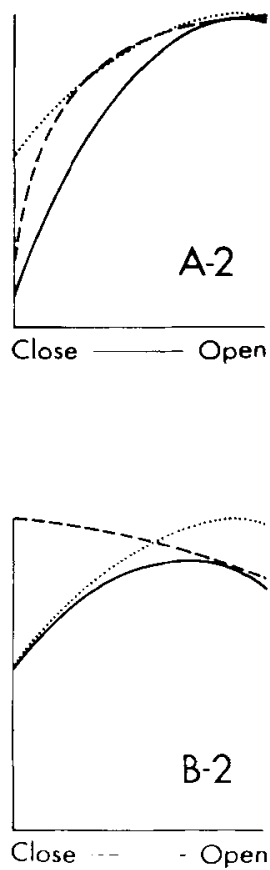

Fig. 7. Trachydosaurus rugosus. Effect of the insertion angle of the muscle on leverage. In $\mathrm{A}$, the fibers insert normally with the jaws agape; in B, the fibers insert normally with the jaws closed. The graphs show moment arm (dotted), an idealized length-tension relation of the sarcomeres (dashed), and the product thereof (solid line) plotted against degree of opening. The ordi- nate is percent of maximum moment. In A-1 and B-1 the plateau region of the individual sarcomeres occurs when the mandibles are gaping; in A-2 and B-2 this occurs when the mouth is nearly closed. The moment observed is the product of these two values, and the force at the symphysis changes accordingly. could generate crossing vectors that would permit the magnitude and direction of load to be controlled.

3) The third explanation for complexity is pinnation. Change of angle permits modification of fiber length, and the angle/length parameters may be matched to variously desirable combinations of force and excursion (or velocity). For instance, it may be possible to reduce the excursion of a muscle and hence the fiber length (while maintaining relative shortening). This does permit more effective packing of sarcomeres, so that a greater proportion thereof will lie in parallel (rather than in series) between origin and insertion; this would increase the force at the cost of a reduction in displacement and velocity. In limb muscles, such reduction of excursion often is facilitated by two-joint arrangements in which two of the three links move in syn- chrony and thus limit the excursion required of the muscle. Muscles may also be shortened by placing them at sites of low excursion. In mandibular attachments, excursion of a muscle may be affected by varying its moment arm, i.e., the normal distance to its line of action. This may be achieved by inserting the muscle more closely to the joint or by insertion at a more shallow angle. (If the fibers cross or curve among sagittal planes, their lengths and angles of insertion must be calculated in three-dimensional space.)

4) The fourth basis of complexity, namely, that it is due to packing factors, requires a brief introductory analysis. One may begin by visualizing a parallel-fibered muscle that connects a simply rotating (one degree of freedom) mandible to the skull (Fig. 8A). In this case, the distance traced (or arc of the circle of rotation) by any insertion site on the 

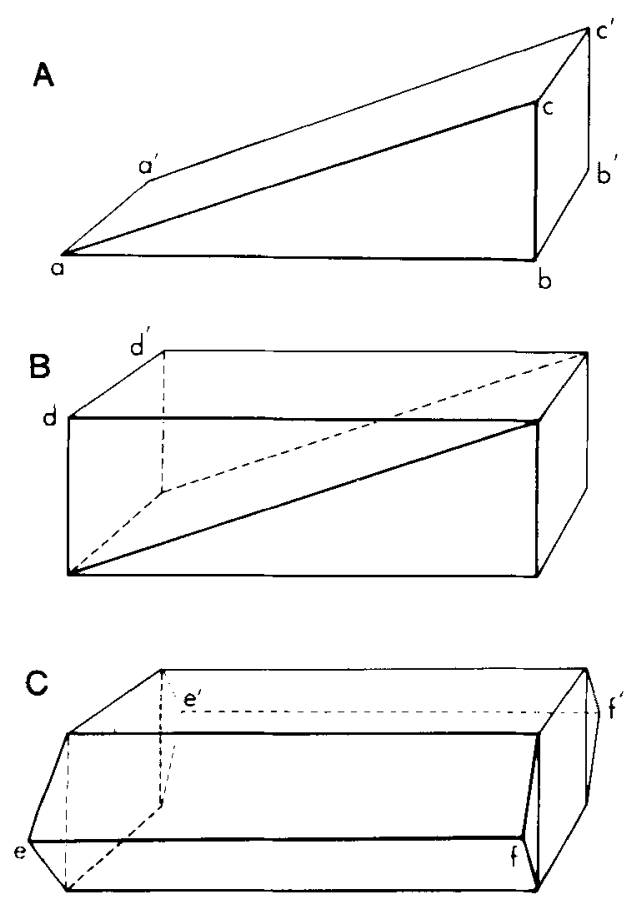

Fig. 8. Theoretical muscle placements in a lizard mandibular system. The assumption is that all sarcomeres are physiologically equivalent. A. Assumption of a single surface each for origin $\left(a, a^{\prime}, c, c^{\prime}\right)$ and insertion $\left(a, a^{\prime}, b, b^{\prime}\right)$, and of all fibers arranged in parallel. Note that the muscle mass will be limited to a simple wedge. B. Utilization of pinnate arrangements, in which the forces are transmitted via aponeuroses, allows an in crease of muscle volume by doubling the volume of the wedge into that of a cuboid. (This may be seen in the arrangement of the main adductors, note Fig. 1.) C. Laterally and medially placed prismatic volumes into which further pinnately arranged fibers may be placed. (The arrangement of the $M$. pterygoideus fits this definition.)

mandible, as well as the straight-line distance (chord) between start and stop of such a site, will be proportional to the distance between the insertion site and the joint around which the mandible rotates. Let us then assume that all sarcomeres of the system will incur equivalent changes in their length-tension relations, that the planes of origin and insertion are simple, and that the connecting fibers lie in parallel (or along segments of concentric circles, Fig. 11). The fiber length (or number of sarcomeres in series) then will be proportional to the distance of the insertion site from the jaw joint. This implies that the number of sarcomeres will

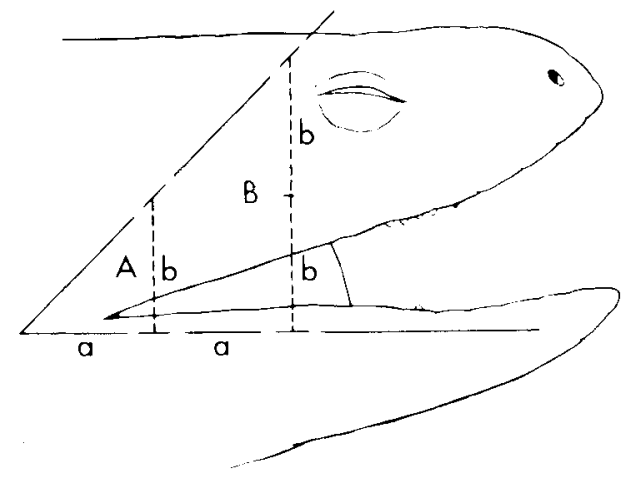

Fig. 9. Fibers of equivalent diameter will produce equivalent force (assuming that their sarcomeres will occupy equal positions on equivalent length-tension curves). Fibers of equivalent diameter will, if placed in parallel, generate moments equivalent to their moment arm; thus, the more distally placed fibers will be more effective; i.e., fiber $B$ will generate twice the moment arm as fiber A, as it inserts at a distance 2a rather than a from the instant center of rotation. However, the number of serially arranged sarcomeres required to form each fiber is also proportional to the moment arm; hence fiber $B$ contains $2 b$ sarcomeres and fiber $A$ only $b$. The moment generated by a sarcomere within $A$ equals $b / a$, and that of a sarcomere within B equals $2 \mathrm{~b} / 2 \mathrm{a}$; consequently, each sarcomere potentially makes an equal contribution within such an array.

increase outward from the joint, in proportion to the distance from the joint to the site of placement. (These statements remain true, independent of the angle at which the fibers of a particular arrangement insert on the mandible.)

Although each such fiber would produce equivalent force, its contribution to the moment acting to rotate the mandible would increase in proportion to the distance between jaw joint and site of insertion. However, fibers with an insertion site farther from the joint must be proportionally longer and hence have more sarcomeres in series. This suggests that the moment generated (and presumably the work done and energy consumed) per sarcomere can be independent of its placement in such a regularly parallel array (Fig. 9).

The described array involves a completely parallel packing of muscle fibers and equal areas for muscle origin and insertion; furthermore, the fibers are packed into a wedgelike space with the apex at the jaw joint (Fig. $8 \mathrm{~A})$. The volume for placement of muscle fi- 

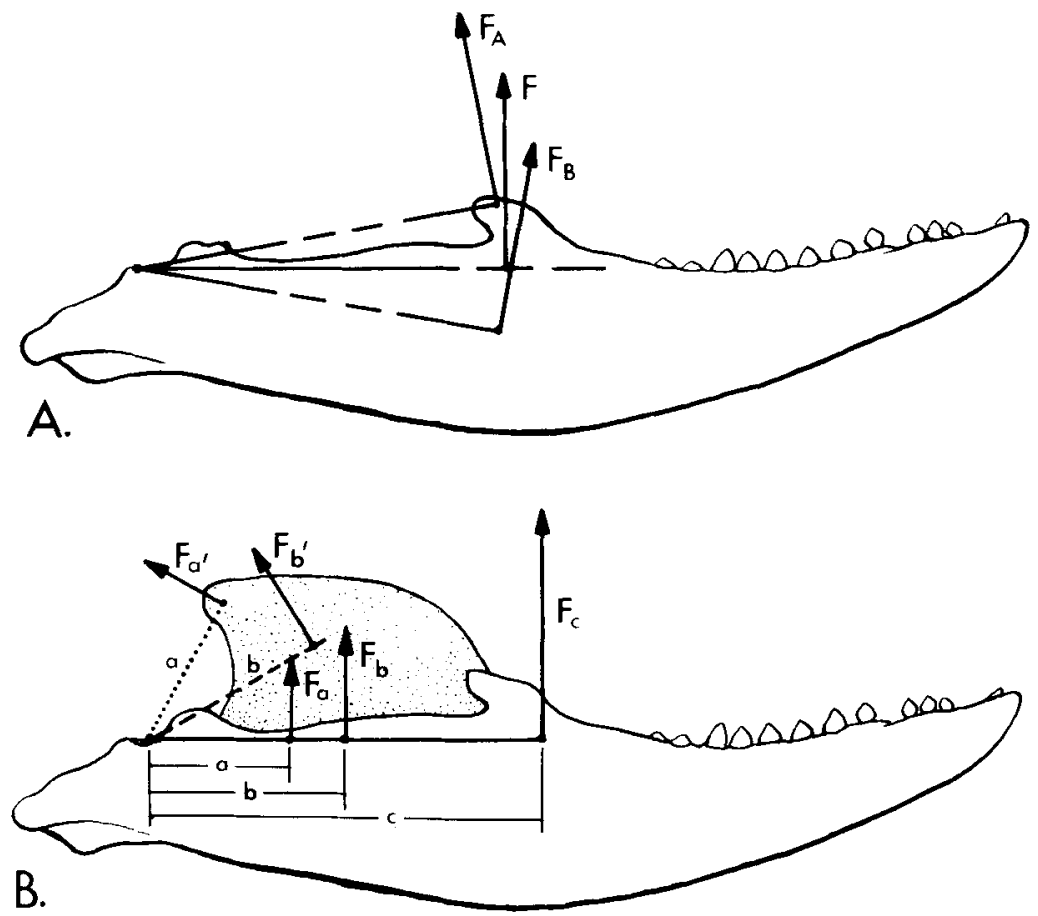

Fig. 10. A. Effect of elevation of the insertion site on the angle to the jaw line of a "normal" muscle. Either the moment arm or the angle of insertion has to be modified with elevation of the insertion site. B. Further complications due to changes in the moment arm of fibers that insert on bony or (in this case) tendinous processes of the mandibular ramus. The fibers of a particular muscle will have parallel length-tension relations assuming constancy of the insertion angle relative to the moment arm and the ratio of fiber-length to moment arm. bers may be expanded by adding spaces that lie outside of the volume of the initial wedge (first as shown in Fig. 8B, and even more as shown in Fig. 8C). Such fibers have to insert via tendons and aponeuroses, likely at a slight angle each, which incurs a variable loss of force at the tendon. However, these allow the volume occupied by the muscle not only to be increased but to be variously shaped.

The assumption that the effective angle of each muscle fiber will be equal implies that the angle of insertion of the fibers (relative to the long axis of the mandible) will have to shift as the attachment site is displaced above or below (or lateral to) the long axis of the mandible (Fig. 10B). This angulation is not the classical pinnation. The additional fibers lie at varying angles to each other, but at a relatively constant angle to their moment arms; hence, the packing does not involve any loss of the force produced by each fiber. (However, the fibers may also be pinnate in the second, i.e., the transverse, dimension, in which case the secondary pinnation involves a loss.)

The insertion angle of any fiber will change as the mandible rotates. However, tendons and aponeuroses will resist tension, but have little resistance to bending and compression. If the fiber inserts on soft tissues rather than the rigid bone, the change of vector may cause the soft tissue system to conform; it will tend to occupy the chord rather than the arc of a circle. Thus the shift of insertion angle may be disadvantageous to the moment arm and to the excursion required to match that of other fibers. In some animals, such as turtles, this problem is compensated for by the development of bony sheaves that 
redirect the forces (Schumacher, 1973), maintaining them in a more advantageous position. Multiply positioned fibers may maintain the aponeurosis in an advantageous position, deflecting it from simple conformation with change of jaw angle. This then remains a usage of complexity.

A final explanation of complexity involves compensation for the change of fiber angle as the movable element (mandible) rotates. It is axiomatic that if the angle to the line between joint and site of insertion is constant and the fiber length is proportional to the length of this line, the fibers will behave equivalently (generate equal moment). Thus, placement of fibers so that their origins lie on one circle, concentric about the jaw joint, and their insertions on another, obviates this problem (Fig. 11). The fiber lengths then may be constant, as will the contribution of each fiber to the moment generated by the muscle. The preceding observations also suggest that change of fiber angles within muscles, often referred to as pinnation, must take into account the position of each set of fibers relative to the center of rotation and to the lines of displacement of the moving elements.

\section{Function of the observed fiber arrangement}

Which of these potential explanations would be applicable to the complex architecture of the masticatory system in Trachy dosaurus?

1) The experimental results differ from the prediction in Wineski and Gans (1984) and furnish no evidence that there are significant differences in the onset and cutoff times or in the contours of the EMG envelopes for the several adductor muscles here discussed. Whereas we have not established independent length-tension curves for the fibers composing the various muscles, the entire adductor musculature appears to be activated almost simultaneously. Exceptions are cases of early activity in adductor muscles that appear to be coactivated with the depressors. However, there is no evidence that the activation of certain portions waxes and wanes independently from that of the whole. This seemingly parallel recruitment of different fascicles does not support the idea of subdivision by temporal activation. Hence this could not explain the complexity of this muscle mass.

2) The cinefluoroscopic (or manipulative) data do not support the concept that the activity of radially arranged bundles provides

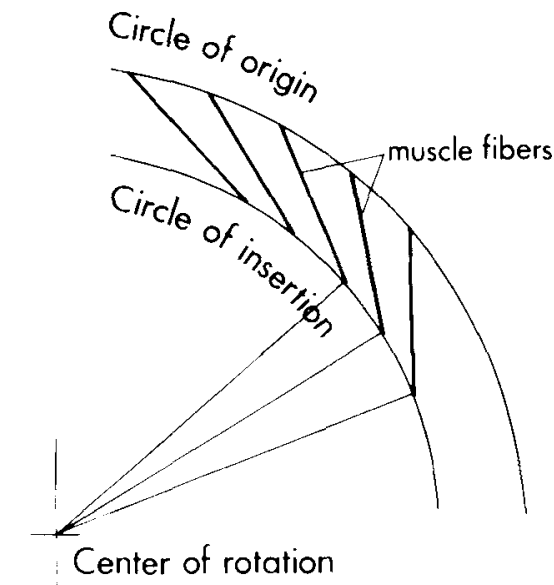

Fig. 11. If the equivalent-length fibers of a mandibular muscle have their origins and insertions on concentric circles, and their sarcomeres are equivalent, the fibers will shorten equivalently during closing. Hence, this is a special condition of Figure $10 \mathrm{~B}$ which is seen in the placement of MAME-3 and MAME-4. Although none of the fibers lie in parallel, the force and moment exerted on the mandible by every such fiber will be equal.

lateral or propalineal shifts of the mandible (even if these were possible in this poorly kinetic skull). Perhaps the simultaneous activation of the mechanically crossed MAME1 and $M$. pterygoideus reduces horizontal forces on the jaw joint (see below on Balance of the Jaw Joint). However, as other muscles are also activated then, the data permit but do not demand this interpretation.

3) The placement of muscles into a shallowangled, low-excursion position demanding many short fibers is clearly seen in the lateral portions of the pinnate MAME-1 (inserting on the quadrate ligament, qalp, of Wineski and Gans, 1984) and the pterygoideus (inserting on the posteroventral and ventrolateral portions of the quadrate). Both muscles lie outside (respectively, lateral and ventral) of the theoretical wedge and pack many short fibers pinnately into a low-excursion array within an otherwise unavailable space (Gans, 1982). Although the angle of pinnation incurs the potential cost of losing some contractile force per fiber, the increase in the number of short fibers between the tendons of origin and insertion substantially increases the amount of force that may act 
on the mandible. The relative shortness of individual fibers reflects the relatively low excursion of the entire muscle.

4) The evidence supports the view that much of the observed complexity serves to facilitate packing of multiple fibers. A simple muscle would be limited to the number of fibers that could originate on a plane running from the supraorbital shelf to the jaw joint and attach on the plane formed by the dorsal surface of the mandible. The volume defined by these planes would contain much less than one-half the volume of the actual adductor mass and would have limited the crushing force severely, both in mastication and in defense.

The fiber arrangement within the MAME$2,-3$, and -4 reflects the earlier consideration of the interactive effects of distance from joint and differential height relative to the tooth row. In all of these muscles, the fibers lie at close to right angles to the line between jaw joint and their particular site of insertion at the time the jaw is closed. The fiber length for each placement appears to be also proportional to the length of the moment arm. This arrangement not only widens the space available for the muscle mass, but also extends it posteriorly, beyond the zone above the mandibular articulation.

It is noteworthy that the posterior fiber mass of the MAME-2 connects to the posterior tip of aponeurosis-Ip. Furthermore, this aponeurosis is constrained to a fixed position, as the scrolled pattern and peripheral attachment of the ligament-Ip and -Iv do not permit much movement relative to the mandible. Indeed, they form an aponeurotic sheet that is stiffened by the mass of muscle (medial MAME-1) it encloses. The flat insertion of the posterior fibers of MAME-2 is likely to keep this sheet in tension along the anteroposterior axis. The arrangement then keeps the fibers in a mechanically advantageous position throughout rotation. The aponeurosis would then represent a pseudocoronoid process, equivalent in action, but not tissue, to the bony coronoids of some mammals and amphisbaenians (Gans, 1981). Clearly, the occurrence of such important soft-tissue structures suggests cautions when reconstructing the musculature of fossils.

Standard descriptions contrast parallel-fibered and pinnate muscles, suggesting that they differ mainly in fiber angle and fiber length, but also assuming that these are constant throughout the array. A more subtle question is raised by mixed systems, such as the MAME-2, the anterior fibers of which are approximately $16 \mathrm{~mm}$ long, although the posterior measure half that. Furthermore, the anterior fibers insert near the level of the coronoid process at approximately a right angle, whereas the posterior ones lie in a roughly horizontal position. In the present case, angle and length of these fibers are matched to their position, reflecting the distance along and angle of the line from the insertion site to the jaw joint. However, the anterior and medial portions of the MAME-2 have a different orientation relative to their insertion/joint line and must shorten differently than do the most posterior fibers, so that the complexity may involve additional factors.

Finally, one notes the constant fiber length seen in the MAME-3 and MAME-4 in which areas of fiber origin and insertion lie concentric about the jaw joint (Fig. 11). Hence the angles of insertion change as the tendon of insertion curves from near vertical (near the coronoid) to almost horizontal; however, fiber length does not change, and the moment arms of all fibers change in parallel with the degree of opening.

\section{Action of the neck muscles}

The dorsal nuchal muscle mass lifts the head, thus permitting further depression of the mandible and facilitating threat displays in a dorsad direction. The more ventral neck muscles apparently act to return the head toward the horizontal, acting in parallel with gravitational force. However, these muscles also fire in synchrony with the adductors whenever the tip of the mandible is forced against the ground during the crushing movement. In so doing they depress the head, and the reaction to their force acts upward against the ventral aspect of the mandible; it is thus synergistic to mandibular elevation and aids the crushing action.

\section{Balance of the jaw joint}

An aspect that deserves more attention relates to the times of activation of the several muscles. Assuming that the records do not indicate cross-talk or the effect of a stretch reflex, the posterior muscles would always seem to fire first, showing a short burst that is almost coincident with the terminal action of the openers. No such action is seen for the shallowly placed MAME-1 or pterygoideus muscles. It may well be that the action of 
these posterior adductors provides a force with a component parallel to the quadrate, but in reverse of that of the openers. This action would load the quadrato-articular joint and reduce the risk that it might disarticulate during wide gape. The more shallowly inserting lateral MAME-1 does not contribute significantly to reseating the mandible; this may account for its silence.

A second point is to note that the vectors of the shallowly inserting $M$. pterygoideus and lateral MAME-1 cross each other, so that the horizontal components of their actions would tend to cancel. These stout muscles generate substantial closing forces, but as large or greater horizontal ones; their simultaneous activation would protect the jaw joint and keep it from overloading in a horizontal direction during closure. This perhaps represents a mechanism analogous to that posited for the $\mathrm{Mm}$. masseter-temporalis cross of mammals which supposedly arose with the development of the temporomandibular jaw joint (Crompton, 1963). The shift of unloading mechanism (from pterygoideus-MAME-1 to masseter-temporalis) may have been forced by the loss of the postarticular insertion site of the old pterygoideus. As the hypothesis is based mainly on osteological materials it deserves experimental test.

\section{Physiological features}

The EMG trains observed during the initial breakage of the snail shell differed from those seen in subsequent reduction bites, both in their duration and in the pulsatile nature of the motor activity, characterized by trains of three to nine pulses at 6-12 (generally $6-8) \mathrm{Hz}$ with a pulse duration of $\overline{\mathrm{x}}=$ $50 \mathrm{msec}$ ( $\mathrm{s}=14 \mathrm{msec}, \mathrm{N}=22$ ). The number of spikes per pulse appeared to increase in most crushing sequences. This pulsatile activation corresponds to reports of similar pulsatile activation of the adductor muscles of Gekko gecko (de Vree, personal communication) and of fishes using pulsatile sequences to crush hard objects (Irish, 1983; G. Lauder, personal communication; all at higher frequencies).

In stimulation experiments, the entire mass of the main adductor was activated at frequencies through and beyond the observed range. The pulsatile frequency noted in this EMG pattern not only produces summation (tetanus) but is the range at which one sees treppe (staircase action leading to unfused tetanus, Houssay et al., 1955). Stimulation at much higher frequencies (those noted for the individual spikes within pulses) produced fused tetanus and rapid fatigue. As the pulses stop once failure occurs, we assume that the applied force likely rose throughout the train; this might reflect only the activation of additional motor units in each pulse. However, the matching of the staircase phenomenon with the pulsatile rate noted in the EMG recordings suggests that each of the multiple spikes recorded during a single pulse reflects a single stimulation in a tetanic train for one of several loosely synchronized motor units.

The mechanism would seem to be well matched to the reduction of hard objects that tend to fail suddenly, then allowing potentially major movements of the jaws. The object is bitten and the jaws are closed with low force, the pulsatile activation starts only after the prey is grasped firmly, limiting impact effects on tooth cusps. The force then increases incrementally, assuming a feedback mechanism. The possibility of significant overshoot and consequent shock on the teeth and central nervous system of the lizard will be limited. It is important to note that this crushing proceeds close to isometrically, as the snail is held firmly between the jaws before the muscles are tetanized; the force increases but the muscles cannot shorten significantly prior to failure of the shell. As the mouth then gapes relatively widely, being near the position for which maximum closing force is generated (Fig. 6), the sarcomeres of the adductor fibers are presumably near the force plateau or "resting position" so that the magnitude of the tetanus represents a multiplier of the maximum isometric force (Fig. 7, B-1).

\section{Ecology}

Under laboratory conditions, Trachydosaurus organisms then represent highly persistent animals that have a clumsy capture mechanism and can crush even hard-shelled food objects once these have been positioned between the tooth rows. The present analysis also has documented that the architecture of their head is permissive of the assumption that selection was for increased force production. However, the analysis, based upon conditions in a single species, cannot provide proof of this. Toward that end we need ecological and comparative information, a point 
generally assumed in analyses based on the techniques of functional morphology, but often left unstated (Gans, 1985). Two sets of questions would seem to be obvious.

The first asks about the actual importance of the forceful bite to the animal. Does it increase deterrence in defense? If so, how often is this important? Does it permit the species to maintain itself in (open?) areas in which less armored and testy animals might incur increased predation? Does it increase the range of food objects taken by this omnivore? How often are hard and large objects taken? Which are they? (Remember that Helix is an introduced species.)

The second set of questions is comparative. How does the mandibular mechanism of Trachydosaurus compare to those of other large lizards, particularly to lizards in equiv. alent environmental circumstances? The morphological comparison should ask both about differences in the concomitants of crushing force and of mandibular mobility. Does the skull differ in architecture, robustness, and breaking strength? Do the muscles differ in architecture and bulk? The ecological comparison should repeat the factors noted in the preceding paragraph. Both of these analyses would seem to represent an obvious project for Australia, as the continent is the home of several groups of large lizards so that comparison may occur both for related species (involving similar genetic background) and more distant ones. The roughly sympatric skinks of the genus Tili$q u a$ would seem to be an obvious test group for the former case. Both groups would offer opportunities for test of hypotheses by field manipulation.

\section{ACKNOWLEDGMENTS}

The Australian work of Gans was supported by a John Simon Guggenheim Memorial Fellowship and a U.S./Australia Cooperative Science Project at the University of Western Australia. He thanks the staff of the Department of Zoology, and particularly its chairman, Professor S.D. Bradshaw, for the gift of specimens, use of laboratory facilities, and hospitality. The present project was funded by DHEW PHS G 1RO1DE05112 and NS 17017-3 and NSF DEB 8121229 (to C.G.) and FGWO 3.9006 .78 (to F.d.V.). We thank the 1983 and 1984 functional morphology seminar groups for assistance, discussion, and comments. G.C. Gorniak kindly commented on an earlier version of the manuscript, and two anonymous reviewers helped us clarify the argument. Parts of this work have been reported at meetings (Wineski, 1982; Gans et al., 1984; Gans, 1984; Gans and de Vree, 1984; and the Australian Herpetological Congress, August 1984, no abstract). The figures were provided by $\mathrm{Mr}$. S. Phippen.

\section{LITERATURE CITED}

Beach, J.C., G.C. Gorniak, and C. Gans 1982 A method for quantifying electromyograms. J. Biomech., 15:611617 (Tech. Note).

Bull, M. 1978 Australian reptile tick Aponomma hydro saurii by host movement. Aust. J. Zool., 26:689-697.

Cogger, H.G. 1983 Reptiles and Amphibians of Australia. Third ed. A.H. and A.W. Reed Pty Ltd., London.

Crompton, A.W. 1963 The evolution of the mammalian jaw. Evolution, 17:431-439.

Gans, C. 1981 Biomechanics. Univ. Michigan Press, Ann Arbor.

Gans, C. 1982 Fiber architecture and muscle function. Exerc. Sport Sci. Rev., 10:160-207.

Gans, C. 1984 Functional morphology as a tool in the study of predator-prey interaction. Program and Abstr. Combined Meet 64th ASIH, 32nd HL, 27th SSAR, Norman, OK, p. 117 (Abstr.).

Gans, C. 1985 Differences and similarities: Comparative methods in the study of mastication. Am. Zool., 25:(in press).

Gans, C., and W.J. Bock 1965 The functional significance of muscle architecture - a theoretical analysis. Ergeb. Anat. Entwickl., 38:115-142

Gans, C., and F. de Vree 1984 Temporal summation in the adductor musculature of the lizard Trachydosaurus: Treppe and tetanus in the crushing of snails. Am. Zool., 24:108a (Abstr.).

Gans, C., and G.C. Gorniak 1982 A simple, transportable, instructional module for initial experiments in functional morphology. The Morphology Teacher (Am. Soc. Zool.), No. 20.

Gans, C., F. de Vree, and D.R. Carrier 1984 Muscle architecture, activity and biological role in the masticatory system of a lizard. Anat. Rec., 208:59A (Abstr.).

Gomez, N.M.B., and J.-P. Gasc 1973 Etude de la biomecanique du mouvement de ferméture de la mandibule chez Ophisaurus apodus (Sauria, Anguidae). Pap. Avul. Zool. Sao Paulo, 27:1-25.

Gordon, A.M., A.F. Huxley, and F.J. Julian 1966 The variation in isometric tension with sarcomere length in vertebrate muscle fibers. J. Physiol., 84:70-192.

Gorniak, G.C., and C. Gans 1980 Quantitative assay of electromyograms during mastication in domestic cats (Felis catus). J. Morphol., 163:252-281.

Gorniak, G.C., H.I. Rosenberg, and C. Gans 1982 Mastication in the tuatara, Sphenodon punctatus (Reptilia: Rhynchocephalia), structure and activity of the motor system. J. Morphol., 171:321-353.

Herring, S.W., A.F. Grimm, and B.R. Grimm 1984 Reg. ulation of sarcomere number in skeletal muscle. A comparison of hypotheses. Muscle Nerve, 7:161-173.

Houssay, B.A., J.T. Lewis, O. Orías, E. Braun-Ménndez, E. Hug, V.G. Foglia, and L.F. Leloir 1955 Human Physiology. McGraw-Hill, New York.

Irish, F. 1983 The dynamics of seed eating in the characiform fish Colossoma. Am. Zool., 23:1027. 
Mackenna, B.R., and K.S. Turker 1983 Jaw separation and maximum incising force. J. Prosthet. Dent., 49:726-730.

Schumacher, G.H. 1973 The head muscles and hyolaryngeal skeleton of turtles and crocodilians. In: Biology of the Reptilia. C. Gans and T.S. Parsons, eds. Academic Press, London, Vol. 4, pp. 101-199.

Smith, K.K. 1982 The functional morphology of the jaw adducting muscles in Varanus exanthematicus. J. Morphol., 173:137-158.

Smith, K.K. 1984 The use of the tongue and hyoid apparatus during feeding in lizards (Ctenosaura similis and Tupinambis nigropunctatus). J. Zool. (Lond.), 202:115-
143.

Throckmorton, G.S. 1978 Action of the pterygoideus muscle during feeding in the lizard Uromastix aegyptius (Agamidae). Anat. Rec., 190:217-222.

Williams, P.E., and G. Goldspink 1971 Longitudinal growth of striated muscle fibers. J. Cell. Sci., 9:751767.

Wineski, L.E. 1982 Mastication in the omnivorous lizard Trachydosaurus rugosus. Anat. Rec., 202:206A (Abstr.). Wineski, L.E., and C. Gans 1984 Morphological basis of the feeding mechanics in the shingle-back lizard Trachydosaurus rugosus (Scincidae, Reptilia). J. Morphol, 181:271-295. 